\title{
Natura@economía
}

ISSN 2226-9479 (Versión electrónica) Website: http://revistas.lamolina.edu.pe/index.php/neu

\section{Estudio Socio Económico para la identificación de oportunidades empresariales. Línea Base del Caserío El Choloque}

\author{
Socio Economic Survey to identify business opportunities. Baseline of Caserío El \\ Choloque. CC Tongorrape
}

Karina M. Yachi Del Pino ${ }^{1 *}$

${ }^{1}$ Universidad Nacional Agraria La Molina, Lima, Perú. Email: yachidelpino@lamolina.edu.pe

Recepción: 15/05/2018; Aceptación: 15/12/2018

\begin{abstract}
Resumen
El objetivo del estudio fue identificar los principales caracteres de la población de El Choloque, a través de la línea base, con la finalidad de detectar las oportunidades empresariales, para ello, se utilizó el método de triangulación (investigador, método cuantitativo y método cualitativo) con la finalidad de obtener resultados confiables. El Caserío "El Choloque", es habitado por 56 familias rurales, que desarrollan actividades como: crianza de animales, agricultura en pequeñas extensiones de tierra. Paralelamente existe un aprovechamiento organizado del bosque seco, a partir de la organización en las asociaciones, siendo la más importante Asprobos (Asociación de Protección de los Bosques Secos). La agricultura les genera ingresos y se complementa con la crianza de animales. El rol de Asprobos ha favorecido el aprovechamiento de sus recursos orientados al desarrollo del valor agregado, la producción de miel y derivados, la elaboración de mermeladas, entre otros, siendo este aspecto una oportunidad de negocio. Finalmente se concluye que, es el poblador un agente capaz de ingresar a mercados competitivos mediante la utilización de sus recursos, es decir de convertirse en generadores de ingreso, este hecho podrá permitir resolver problemas que actualmente la población enfrenta, asegurando la sostenibilidad de sus recursos y del aprovechamiento de oportunidades vinculadas a la generación de ingresos.

Palabras clave: línea base; participación; desarrollo local; oportunidades; negocios; economía rural.
\end{abstract}

Forma de citar el artículo: Yachi, K. 2018. Estudio Socio Económico para la identificación de oportunidades empresariales. Línea Base del Caserío El Choloque. Natura@economía 3(2):75-89(2018).

DOI: http://dx.doi.org/10.21704/ne.v3i2.1522

* Autor de correspondencia: Yachi, K. Email:yachidelpino@lamolina.edu.pe

(C) Facultad de Economía y Planificación, Universidad Nacional Agraria La Molina, Lima, Perú. 


\begin{abstract}
The objective of the study was to identify the main characters of the El Choloque population, through the baseline, in order to detect business opportunities, for this, the triangulation method (researcher, quantitative method and qualitative method) was used. in order to obtain reliable results. The "El Choloque" hamlet is inhabited by 56 rural families, who carry out activities such as: raising animals, agriculture in small areas of land. At the same time, there is an organized use of the dry forest, based on the organization in associations, the most important being Asprobos (Association for the Protection of Dry Forests). Agriculture generates income for them and is complemented by raising animals. The role of Asprobos has favored the use of its resources aimed at the development of added value, the production of honey and derivatives, the production of jams, among others, this aspect being a business opportunity. Finally, it is concluded that the population is an agent capable of entering competitive markets through the use of their resources, that is to say, of becoming income generators, this fact may allow solving problems that the population currently faces, ensuring the sustainability of their resources and taking advantage of opportunities related to income generation.
\end{abstract}

Keywords: baseline; participation; local development; opportunities; businesses; rural economy.

\section{Introducción}

Determinar la situación socioeconómica de la población objetivo es un punto de inicio, genera información objetiva que permitirá tomar decisiones efectivas, las diversas herramientas que buscan impulsar la generación de ingresos u oportunidades de desarrollo comunitario parten de una evaluación de impacto de los fines y resultados propuestos.

En este sentido, el estudio analiza la situación ex - ante de la población objetivo del proyecto, para poder posteriormente realizar una adecuada medición de los impactos del mismo, incluso al momento de diseñar propuestas que se realicen bajo lineamientos realistas. El trabajo se desarrolla en fases o secuencias (usando herramientas cuantitativas y cualitativas, procesadas mediante técnicas estadísticas) para medir los componentes: Social, Infraestructura, Económico, Productivo, Empresarial, Político - Organizacional y Ambiental, utilizando la matriz de indicadores.

Las condiciones de la población objetivo y su potencial incursión en mercados altamente competitivos requiere de una exhaustiva identificación de las características presente en la comunidad como potenciales emprendedores. Desde la experiencia de Navaro, King, Ortegón, y Pacheco en el "Estudio Línea Base" del Instituto Latinoamericano y del Caribe de Planificación Económica y Social (ILPES) realizado en el 2006 se desprende que: los estudios ex - ante como el diagnóstico socio - económico de la realidad que enfrentan diversas comunidades, involucran la sistemática recolección y presentación de datos para dar una descripción clara de una situación particular en cuando relacionan: ¿Qué? ¿Quién? ¿Dónde? ¿Cuándo? ¿Por qué? ¿Cómo? y normalmente cubren solo una muestra de la población. Sin embargo, si el estudio de línea de base cubre la población en su totalidad, recibe el nombre de censo.

La búsqueda de información cuantitativa se ve favorecida para una mejor profundidad de la explicación con la información cualitativa, y sobre esta última se ve en la práctica un mayor resultado de experiencias de técnicas útiles como las IAP (Intervención 
Acción Participación) afines al diagnóstico participativo útil en el sector rural

El Diagnóstico Participativo Rural, apoya un estudio de línea de base generando información sobre los niveles de conciencia, conocimiento, actitudes y prácticas de una población dada, sobre los temas seleccionados, en un área geográfica específica de modo participativo (Eguren et al., 2005).

Los resultados de los diagnósticos socio - económicos van a tender puentes para el logro del desarrollo humano como es un proceso que permite aumentar y mejorar las capacidades, elecciones y oportunidades de la gente, especialmente aquellas de la población rural y de los pobres, para llevar una larga vida, saludable y plena. Este proceso incluye la expansión de la capacidad de la población y de sus habilidades para ganar acceso y control de los factores que afectan las necesidades esenciales y básicas en sus vidas.

Los componentes del estudio línea base mencionados anteriormente operan desde el recojo de datos sobre necesidades presentes en la población objetivo, en los asuntos políticos, económicos, sociales tanto de sus sociedades como de sus naciones.

En tal sentido, el objetivo del estudio fue proporcionar elementos cuantitativos y cualitativos para determinar las actuales condiciones de desarrollo socio - económico de la población de Choloque, para generar una línea base que permita incorporar los proyectos de investigación sobre el bosque seco, vinculados a los intereses y necesidades de la población en términos de la búsqueda de oportunidades para contribuir a la mejora de sus condiciones socio-económicas.

\section{Materiales y métodos}

El estudio se realizó en la Comunidad Campesina de Tongorrape, Caserío de El Choloque, distrito de Motupe, Perú. Mientras que, el procesamiento de datos se realizó en las instalaciones de la UNALM. La investigación fue tipo descriptiva, buscó identificar los caracteres presentes el caserío de El Choloque. Los datos recabados fueron de tipo cuantitativo, se obtuvieron a través de encuestas realizadas a una muestra poblacional representativa del universo poblacional de El Choloque, y en algunos temas para lograr profundidad se valoraron los de tipo cualitativo y se procesaron hasta obtener información confiable.

En la etapa de producción, procesamiento, de la información recopilada se sistematizó, clasificó y organizó en cuadros, para luego ser procesadas en diversos software como: CS pro 4.01 para la digitación de los cuestionarios, el SPSS 20 y Minitab para el procesamiento de datos, el Excel 2012 para armar los indicadores, y el Microsoft Word 2010 para redacción de los diferentes informes, entre otros. De manera que se disponga de información procesada sobre los caracteres que describen de manera precisa a la población de El Choloque.

\section{Población y muestra}

La muestra del presente estudio, se estimó a partir de 56 unidades familiares, siendo el tamaño de la muestra probabilística, 34 familias, un nivel de confianza de 95, el error estimado del $11 \%$, la selección de las familias fue ejecutado mediante el muestreo aleatorio simple estratificado, buscando la representatividad de la población en función de la distancia geográfica a un punto de referencia (el centro comunal) llegando a abarcar todo el territorio de El Choloque (Dirección Regional de Trabajo y Promoción del Empleo Lambayeque, 2011).

$$
n=\frac{z^{2} \times P \times Q \times N}{e^{2}(N-1)+z^{2} \times P \times Q}
$$

Dónde: Z, Porcentaje de fiabilidad deseado para la media maestral. P, Porcentaje de veces que se supone que ocurre un fenómeno en la población. Q, Porcentaje de no ocurrencia del fenómeno $(1-\mathrm{p})$. e, Nivel de error máximo permitido para la media. 


\section{Resultados y discusión}

En los últimos cinco años El Caserío de Choloque ha tenido un gran desarrollo económico. La provincia de Lambayeque presenta un performance empresarial dinámico (Tabla 1).

Tabla 1. Tabla Performance Empresarial en la Provincia de Lambayeque

\begin{tabular}{lcc}
\hline \multicolumn{1}{c}{ Detalle } & $\mathbf{2 0 0 7}$ & $\mathbf{2 0 0 8}$ \\
\hline Microempresas Constituidas & 101 & 149 \\
Talleres Desarrollados & 42 & 52 \\
\hline N $^{\circ}$ Participantes en talleres & 947 & 1776 \\
\hline Sectores Microempresas & 101 & 149 \\
Industria & 3 & 17 \\
Comercio & 27 & 55 \\
Servicios & 70 & 68 \\
Construcción & $\ldots$. & 6 \\
Agropecuaria & 1 & 1 \\
Tercerización & $\ldots .$. & 2 \\
Modalidad Empresarial & 101 & 149 \\
E.I.R.L. & 32 & 72 \\
\hline S.R.L. & 39 & 32 \\
S.A.C. & 30 & 44 \\
S.A. & $\ldots .$. & 1 \\
Capital & 676 & 1183 \\
Bienes Dinerarios & 713 & 135 \\
& 226 & 529 \\
Bienes no Dinerarios & 860 & 036 \\
& 449 & 657 \\
\hline
\end{tabular}

Fuente: Dirección Regional de Trabajo y Promoción del Empleo Lambayeque (2011).

El poblador del Caserío de Choloque, no cuenta con registros confiables sobre el caserío específicamente, sin embargo, se hallaron estudios sobre el distrito de Motupe, siendo este el punto de partida. Está conformado por dos comunidades campesinas: Santa Julia y Tongorrrape. Se llegó a identificar los principales caracteres descriptores del distrito con la finalidad de identificar algunos patrones de comportamiento y rasgos estructurales útiles para la investigación. El distrito de Motupe fue creado por Ley 12419 el 7 de noviembre de 1455 .

La economía del distrito de Motupe es dinamizada principalmente por los agro negocios que se sustentan en los sectores agrario e industrial, complementado por el sector servicios básicamente centralizado en la capital. Una importante expresión de esta situación es la composición de la población económica activa (PEA) del distrito: de cada 10 trabajadores, 3 a 4 son del sector agrario, 1 a 2 del sector industrial, 2 a 3 del sector servicios, 1 a 2 buscan trabajo. La economía local presente rasgos predominantes como la desarticulación de las organizaciones de productores y la debilidad de gestión productiva-comercial de estas, lo que constituye el proceso crítico que genera menor productividad y mayores costos - por tanto, menor nivel de rentabilidad de sus actividadespor la precaria y dispersa capacidad de negociación individual. Asimismo, ocasiona la pérdida de oportunidades aprovechables que requieren organización económica de los productores tales como articulación a cadenas productivas, inserción a los mercados especiales como los orgánicos.

La Agencia Agraria de Motupe no lleva registros detallados de la producción de los caseríos por la dificultad en la obtención de la información, la falta de personal y otros aspectos institucionales.

Se identificó la consolidación del mango como producto líder del desarrollo económico de la zona, este hecho se explica en su crecimiento sobre otros tipos de cultivos, en las campañas se observa un, crecimiento sostenido del volumen de producción de alrededor de 2,500 a 4,520, hasta llegar a 47,033 toneladas mayormente de la variedad Kent, más resistente a la travesía, menos susceptible a la deficiencia de calcio que las variedades Haden y Tomy Atkins, cuyo destino principal es la exportación. Dándose generación de empleo 
permanente y temporal agrícola, impulso a la reactivación de los servicios vinculado al acopio y transporte a Piura (Tambogrande Sullana) para su tratamiento, clasificación, empaque en el Puerto de Paita (vía marítima).

El menor nivel y calidad educativa de un importante sector de los pequeños productores/as limita al despliegue de sus talentos y facilita la generación de una baja autoestima y la desconfianza. La capacitación y asistencia técnica mediante programas dirigidos a los productores/ as adultos/as incorporando a los/as hijos/ as jóvenes, frecuentemente casi no existe lo que dificulta superar las debilidades de gestión predial y organizativa.

Respecto a otras actividades rurales, existe vulnerabilidad de las economías dependientes de la crianza pecuaria extensiva: la variabilidad del clima, afecta a la ganadería sensible a la menor producción de pastos o de algarroba, especialmente por la mayor frecuencia de ocurrencia de años de sequía o la campaña con extrema pluviosidad que afecta la producción de algarroba (por la caída de la floración).

\section{La actividad productiva de la zona}

La creciente producción agraria- agrícola, ganadera y forestal- se desarrolla en dos agro ecosistemas típicos - el valle y los bosques seco- en una superficie estimada alrededor de 37 mil 183 has, de las cuales el 22\% son terrenos de uso agrícola principalmente bajo riesgo, y el $78 \%$ son bosques, montes y pastos, en condiciones de secano, de uso ganadero, apícola y forestal principalmente.

Las unidades agrarias ocupan alrededor de 50 mil has del distrito, las que están constituidos por las siguientes clases de productores: a) Dos comunidades campesinas, cuyo territorio de uso agrario ocupa el $71 \%$ de la superficie, de acuerdo al CENAGRO, básicamente constituido por bosques secos y pastos; Unas 1,200 pequeñas unidades agrarias menores de 10 has, ocupan el $11 \%$ de la superficie, siendo 4,5 has el tamaño de una unidad promedio; Otras 313 medianas unidades, con áreas entre 10 a menos de 20 has, ocupan el $8 \%$, siendo 12,7 has del tamaño de la unidad promedio; $\mathrm{y}$, un total de 120 propiedades mayores de 20 has ocupan el $9 \%$, siendo 83 has el tamaño de la unidad promedio. Sus unidades de pequeña propiedad son de posesión familiar, las mismas que constituyen fuente de autoempleo familiar y empleo eventual.

Es importante destacar el rol de los bosques secos, siendo uno de los principales el de algarrobo, del que se explica la dinámica económica que gira alrededor del aprovechamiento de los productos del bosque: algarroba, madera y pasto; temporalmente pequeñas áreas de uso agrícola con cultivo de maíz amiláceo. La tala creciente del bosque para uso doméstico (leña) pero la mayor extracción está destinada al comercio mayorista de leña y carbón. Recientemente, la pequeña actividad agroindustrial domestica tal como producción de miel y derivados, así como de algarrobina.

\section{Usos y fines de la tierra}

En el estudio de la agricultura de la costa peruana realizado por Eguren (2005) se encuentra disparidad de condiciones de la tierra, resultando que existe distinto grado de uso de la tierra en los valles del norte. Hay una relación inversa entre la extensión total de tierras bajo riego de que dispone cada valle de la costa y la proporción que se mantiene de tierras cultivadas, la mitad del área cultivable del valle ChancayLambayeque puede mantenerse libre de siembras por falta de agua (INEI, 1993). El uso de la tierra se eleva conforme es mayor el volumen de recursos hídricos. El uso de la tierra en Choloque es: agrícola $(72,86)$, forestal $(17,08)$, pecuario $(6,82)$ y otros $(3,25)$. 
Tomando datos históricos, entre 1972 y 1994 el último censo registra un aumento de unidades agropecuarias en la costa, pasando de 126000 unidades agropecuarias en 1972, a 2260008 un aumento de cerca de $80 \%$. En ese mismo periodo, el área agrícola de la región solo aumento 9,8\% (INEI, 2007).

El censo de 1994 (INEI, 207a) registra una extensión de 836000 hectáreas con riego. La mayor parte de esta superficie $(52 \%)$ se localiza en la costa norte; algo más de un tercio (35\%) en la costa central, y el resto $(8 \%)$ en la costa sur. Es importante destacar que se toma información de los departamentos de Lambayeque y de otros cuatro situados en la costa norte del país.

En este sentido se entiende que la percepción de la población posiciona a la agricultura como actividad principal, sin embargo, el uso de las tierras para fines agrícolas está asociado a la existencia de riego entre otros factores, existiendo en la zona recursos naturales valiosos: los bosques secos sobre los cuales hoy se gestionan mecanismos de protección a través de distintas prácticas: El Plan de Manejo Forestal desarrollado por Asprobos es uno de los más interesantes en la zona (Asprobos, 2004).

Las extensiones de tierra en el Caserío de Choloque giran en torno a la pertenencia comunal $(38,5 \%)$ y propia $(55,8 \%)$, la última es únicamente sobre las de uso agrícola y de vivienda, al desarrollar el uso forestal este se realiza sobre tierras comunales que son las de mayor significancia en amplitud 487 ha, en las se distinguen tres categorías de bosque.

El bosque seco de colina (cuya área es el 55\%), el bosque seco ralo de llanura y el bosque seco ralo de lomada, además en la zona se encuentra matorrales formaciones vegetales cuya extensión es de 60 ha (Tabla 2).
Intentando profundizar algunos aspectos sobre las tierras, interesa identificar ¿cómo fueron adquiridas? Información difícil de obtener por las resistencias de la población para mencionar el origen de las tierras de las cuales hoy realizan un aprovechamiento agrícola y forestal, este hecho se explica en la respuesta hallada en diversas entrevistas en las que el factor común es el asentamiento de familias de otras zonas en busca de mejoras llegando así a la Comunidad Campesina de Tongorrape.

Los recursos del bosque se pueden aprovechar durante todo el año, por su propia naturaleza, los principales productos son frutas $(67,65 \%)$, destacando el sapote para alimento de los animales, la miel $(14,71)$ que puede ser de algarrobo u miel orgánica sobre la que tienen un sistema de producción estructurado en la parte más alta del bosque, los restos de madera como el palo santo cuyo aroma es utilizado como ambientador entre otros.

La intervención en el bosque actualmente es de forma sostenible, se han logrado controlar varios problemas de explotación y destrucción, sin embargo, los árboles presentan varios tipos de enfermedades, en el recorrido de campo se diagnosticó en una muestra de árboles la presencia de enfermedades ocasionadas por Ganoderma $s p$ el cual causa la pudrición de medula de las especies leñosas. Si bien es cierto,
Tabla 2: Extensión de las tierras del caserío El Choloque- Sector El Cardo. CC de Tongorrape

\begin{tabular}{llc}
\hline \multicolumn{1}{c}{ Zona } & \multicolumn{1}{c}{ Tipo de Tierras } & $\begin{array}{c}\text { Áreas } \\
\text { (ha) }\end{array}$ \\
\hline Loma Diana & Bosque Seco de Llanura & 95 \\
Loma Facunda & Bosque Seco de Lomada & 110 \\
Hualtacal - Charanal & Bosque Seco de Colina & 212 \\
Uveral & Formación Vegetal & 60 \\
Agrícola y Pecuaria & Predios Familiares & 157 \\
\hline Total & & 634 \\
\hline Fuente: Asprobos (2004). & &
\end{tabular}


son habitantes naturales de los bosques, en un futuro con el sobre pastoreo y daños mecánicos ocasionados sobre los árboles, se podría llegar favorecer la diseminación de la enfermedad. Otra de las enfermedades detectadas es la alternaría, que ocasiona la antracnosis, rota y cierra su ciclo durante todo el año alternando con hospederos secundarios del bosque. Para el cual se recomienda incluir en el manejo del cultivo a los cercos para reducir la presencia de inoculo infeccioso en las plantaciones cercanas a los frutales (Llacsahuanga \& Venancio, 2011).

Incluso se identificaron plagas que pueden causar muchos perjuicios para cultivos como el mango y cítricos, como la mosca de la fruta que se presenta como principal problema para los productores, esta plaga encuentra refugio y alimento suficiente en el bosque de especies que proveen de polen y azucares todo el año.

Las poblaciones de la mosca de la fruta cierran su ciclo, y pasan desapercibidas para los pobladores, incrementado el número de individuos cuando las frutas alcanzan su madurez y punto de cosecha, lejos de los controladores naturales del bosque las poblaciones de mosca crecen exponencialmente. Por lo que, se recomendó monitorear la dinámica poblacional con trampas caseras y controlar con productos naturales como el spinosad, de nombre comercial GF-120 utilizado en la certificación orgánica.

Un tema alarmante es la falta de información sobre los productos del bosque seco, es decir que el poblador solo hace una recolección espontanea; sin embargo, aún no aprovecha el potencial de los recursos del bosque y se observa en las ramas secas de varias variedades de árboles que emana un aroma agradable, demostrando un alto valor para la aromaterapia como oportunidad de negocio. El poblador ha identificado en la miel un espacio de desarrollo, el impulso de Asprobos está logrando el desarrollo de algunos derivados, sin embargo, solo están presentes en un mercado local, sus condiciones de producción y la certificación ya obtenida la convierte en un producto con gran potencial considerado además en el mercado regional y nacional

Asprobos utiliza la miel colectada por las abejas, hormigas y avispas, es decir que le dan utilidad a los recursos del medio explotando diversas formas artesanales de recolección como las pequeñas cajas de crianza de abejas y avispas.

\section{Empresa y organización}

Asprobos como asociación ha incursionado de forma colectiva en la actividad empresarial, cuentan con clientes de sus productos en Chiclayo, y están en la búsqueda de tener puntos de venta en Lima. Sus precios son: Miel Orgánica (400 g * $\mathrm{S} / 20,00)$, Miel de Aspargate (250 g* S/ 60,00), Mermelada de Mamey $(250 \mathrm{~g} * \mathrm{~S} /$ $4,00$ y $600 \mathrm{~g} * \mathrm{~S} / 10,00)$.

\section{Índice de desarrollo humano (IDH)}

En la presente investigación se toma como parámetro de evaluación el índice de desarrollo humano (IDH) de la provincia de Lambayeque, en el que se contempla caracteres que van más allá del enfoque monetario. El IDH, presenta tres aspectos fundamentales: "ingresos que permita una vida decente; educación que auspicie conocimientos para la toma de decisiones adecuadas; y salud que les permita tener una vida saludable y productiva".

El estudio de la línea base de El Choloque requiere del uso de IDH, sin embargo, no existen estudios previos de la zona y es relevante realizar un comparativo con IDH existentes porque se puede recoger muestras de desigualdad, haciendo posible un mejor diagnóstico de la realidad social de la zona más próxima, en tal sentido se toma los 
datos de la provincia y de la región para posteriormente llegar a conclusiones de las condiciones socio - económicas de la zona (Diagnóstico de la Micro Región Motupe, 1992).

Medir comparativamente los indicadores existentes sobre la región y sobre la provincia de Lambayeque, fue posible contando con la referencia de los datos elaborados por el PNUD y el Banco Central de Reserva del Perú, datos que permiten comprender diversos aspectos de la realidad de El Choloque y su población.

La región Lambayeque está ubicada en la costa norte del país y abarca una superficie de 14,2 mil kilómetros cuadrados. Conformada por tres provincias - Chiclayo, Ferreñafe y Lambayeque - alberga a 1,1 millón de habitantes, que representan el $4,1 \%$ de la población del país. Su capital Chiclayo, de gran movimiento comercial, concentra más de la mitad de la PEA (población económicamente activa) ocupada de la región. La ubicación estratégica de Chiclayo la convierte en punto de encuentro de diversos agentes económicos que provienen tanto de otras ciudades costeñas como de la sierra y la selva. Por ello, el comercio es uno de los sectores principales, representando el $25 \%$ de la actividad económica de la región. Respecto a la provincia de Lambayeque se ubica el ranking 44 de 198 provincias evaluadas en el IDH Perú 2005 (Tabla 3).

La región Lambayeque ocupa el sétimo lugar en el ordenamiento regional del IDH 2005 después de Callao, Lima, Tacna, Ica, Arequipa y Moquegua. El ordenamiento según provincias (con relación al total de provincias del país) estaría mostrando disparidades al interior de la región; así, mientras Chiclayo ocupa el orden 19 entre 198 provincias, Ferreñafe ocupa el puesto 61.

En términos de niveles de renta, la región Lambayeque aún muestra un importante retraso respecto al promedio nacional: el valor de la producción per cápita al 2007 fue un $69 \%$ del promedio nacional y un $47 \%$ del promedio de Lima. Esta participación es incluso ligeramente menor a la que tuvo la región a inicios de la década.

Tabla 3: IDH Provincia de Lambayeque 2005

\begin{tabular}{|c|c|c|}
\hline \multicolumn{3}{|c|}{$\begin{array}{l}\text { Índice de Desarrollo Humano Prov. } \\
\text { Lambayeque }\end{array}$} \\
\hline \multicolumn{2}{|c|}{ IHD 2005} & Lambayeque \\
\hline \multirow{2}{*}{ Población } & Habitantes & 258,747 \\
\hline & Ranking & 15 \\
\hline \multirow{2}{*}{ Índice de DDHH } & IDH & 0,6047 \\
\hline & Ranking & 44 \\
\hline \multirow{2}{*}{$\begin{array}{l}\text { Esperanza } \\
\text { vida al nacer }\end{array}$} & Años & 89,9 \\
\hline & Ranking & 72 \\
\hline \multirow{2}{*}{ Alfabetismo } & $\%$ & 89,9 \\
\hline & Ranking & 72 \\
\hline \multirow{2}{*}{ Escolaridad } & $\%$ & 80,9 \\
\hline & Ranking & 131 \\
\hline \multirow{2}{*}{$\begin{array}{l}\text { Ingreso familiar } \\
\text { per cápita }\end{array}$} & N.S. Mes & 403 \\
\hline & Ranking & 30 \\
\hline
\end{tabular}

Fuente: PNUD (2005).

1/ Mientras más cercano el índice a la unidad mayor desarrollo humano relativo.

2/ Orden ocupado entre 198 provincias existentes en el país.

Lambayeque es relativamente pequeño en términos económicos; representa cerca del $2,7 \%$ de la producción nacional y es la octava economía a nivel nacional si se mide por el valor bruto de la producción del año 2007. Esta posición relativa la obtiene aun cuando en términos de superficie, con una extensión de $14231 \mathrm{Km}^{2}$, es la tercera región más pequeña del Perú luego del Callao y Tumbes. Ello refleja el potencial productivo de la región, pues ocupa el segundo lugar si se tiene en cuenta el valor bruto de producción por $\mathrm{km}^{2}$ (Banco Central de Reserva del Perú, 2004). "Región Lambayeque: Cifras y reflexiones para el debate".

Losprincipales rasgos de la población 
rural de la provincia de Lambayeque son: población rural $(48,3 \%)$, vivienda con electricidad (49,8\%), Vivienda con agua $(43,0 \%)$, analfabetismo $(9,4 \%)$; con primaria $(94,8 \%)$ y con secundaria (76,1\%) (Encuentro Económico Región Lambayeque, 2004).

En el Caserío de Choloque se identifican características sobre su conformación como población, partiendo del número de familias, las que se mencionaron en un inicio: 56 unidades cuya procedencia es heterogénea, ya que existen pobladores de otros departamentos.

En el poblado predomina una conformación de familia joven con hijos aun en las escuelas en su mayoría, los cuales demuestran un alto sentido de búsqueda de mejoras, tienden a colocar sus aspiraciones en la formación educativa, la capacitación y la actividad organizada para crear ingresos, así lo demuestran el total de organismos presentes para buscar soluciones a sus problemas, como: Asprobos (Asociación de Protección de los Bosques Secos), la Comisión de Regantes del Sub sector de Riego Tongorrape, La
Junta de Administración Local El Cardo, La Asociación de Productores Orgánicos de Tongorrape, La Ronda Campesina de Choloque, las Asociaciones de Padres de Familia de Cerquen, Del Carmen y del IE Quiñones; con los que hay una interacción directa además de estar vinculados territorialmente con la Comunidad Campesina de Tongorrape de la cual son parte. Es en este escenario, el jefe del hogar es masculino, se desarrolla como agricultor con estudios a nivel primaria y en algunos casos con secundaria incompleta, esta es una realidad común a la mayoría de las familias del caserío, sin embargo, también se nota la presencia de algunos pobladores con estudios técnicos.

El nivel educativo del ama de casa, es inferior al del jefe de hogar; sin embargo, todos los hogares tienen inscritos a sus hijos en escuelas e institutos de formación, las escuelas se encuentran en otros caseríos y en el cruce de Tongorrape, los estudios superiores necesariamente se desarrollan en Motupe y Chiclayo, solo en algunos casos estos se dan en Lima (Tabla 4).

Tabla 4: Rasgos del jefe de hogar, grado de instrucción y ocupación

Nivel de educación alcanzado

Jefe (a) de la Familia

Sin Primaria Primaria Secundaria Secundaria Técnico Técnico estudio Incompleta Completa Incompleta Completa Incompleto Completo Universitario

\begin{tabular}{|c|c|c|c|c|c|c|c|c|c|}
\hline \multirow{7}{*}{$\begin{array}{l}\text { Ocupación } \\
\text { principal }\end{array}$} & & $\%$ & $\%$ & $\%$ & $\%$ & $\%$ & $\%$ & $\%$ & $\%$ \\
\hline & Agricultor & 5,88 & 14,71 & 23,53 & 14,71 & 5,88 & 0,00 & 5,88 & 0,00 \\
\hline & $\begin{array}{l}\text { Productor } \\
\text { pecuario }\end{array}$ & 0,00 & 0,00 & 0,00 & 0,00 & 0,00 & 0,00 & 0,00 & 0,00 \\
\hline & Ama de casa & 2,94 & 2,94 & 8,82 & 0,00 & 0,00 & 0,00 & 0,00 & 0,00 \\
\hline & Comerciante & 0,00 & 0,00 & 0,00 & 0,00 & 2,94 & 0,00 & 0,00 & 0,00 \\
\hline & Otro & 2,94 & 0,00 & 0,00 & 0,00 & 0,00 & 0,00 & 0,00 & 0,00 \\
\hline & No contesta & 2,94 & 5,88 & 0,00 & 0,00 & 0,00 & 0,00 & 0,00 & 0,00 \\
\hline
\end{tabular}

Fuente: Plan de Desarrollo Económico Local Motupe (2010). 
En el hogar, las familias jóvenes mantienen viviendas construidas con materiales de la zona, existe un uso general del adobe, quincha y madera en paredes puertas y ventanas; sin embargo, las casas en su mayoría no cuentan con las instalaciones acabadas, sin servicios básicos ni comodidades. Lo que predomina en los pisos es la tierra, se encontraron casas de un solo piso, con corrales de animales menores y cercos alrededor de las habitaciones, su ubicación en general está cerca de fuentes de agua como ríos y riachuelos, las mismas se encuentra dispersas en todo el territorio del caserío (Tabla 5).

La vivienda típica en el Caserío de El Choloque, es de propiedad de la familia que la habita, el número máximo es de ocho, es decir que en promedio son casas de tamaño mediano con cinco habitaciones destinadas a diferentes fines, en donde el tipo de fuente para la iluminación es la vela; los servicios higiénicos están fuera de la casa, este rasgo es típico en las zonas rurales. Existe el uso de pozos sépticos y similares, como servicio higiénico es decir que su nivel de bienestar es limitado por la ausencia de agua canalizada a través de tuberías y finalmente la falta de desagüe, por lo que no pueden desarrollar prácticas higiénicas adecuadas, sin embargo, han delimitado ciertas zonas para estos fines (Tabla 6 y Tabla 7).

Los cultivos que la población de El Choloque desarrolla son principalmente para cubrir necesidades de alimentación, la crianza de animales menores es complementaria, en la zona es notoria la presencia de las menestras, algunos frutos y del maíz, en el valle la participación del maíz es alta al año 2012 en Tongorrape la producción de maíz alcanzo 1,607 toneladas por campaña, cuyo precio en chacra está en 0,75 céntimos de sol, y cuyos rendimientos promedios son $5781 \mathrm{~kg} /$ ha (según fuente de la Oficina Agraria de Motupe).
En segundo lugar, de importancia de los cultivos del Caserío se encuentra al Mango, el mismo que solo en la Comunidad Campesina de Tongorrape ha dejado para el 2012 una producción de 1925 toneladas, variedad Kent, cuyo precio en chacra es de 1,08 nuevos soles y los rendimientos son $6754 \mathrm{~kg} / \mathrm{ha}$. Es decir que la inclinación por estos cultivos es similar en términos generales al comportamiento de toda la CC de Tongorrape. Los Aspectos Económicos describen a una población que puede generar ingresos de subsistencia a través de sus prácticas cotidianas, aún presentan debilidades para el ahorro y la inversión, ya que esta depende de la generación activa de ingresos. La población está sujeta a cubrir necesidades, sin que esto limite el desarrollo de algunas actividades de fortalecimiento de sus capacidades como la superación de obstáculos a través de grupos organizados, aún con un entorno poco alentador porque es desolador el estado de sus caminos y la irregularidad de sus medios de transporte.

En la Tabla 8, se muestra la generación de ingresos de cada familia, siendo la tarea del jefe de familia aportar el $90 \%$ del ingreso total, pero si se observan los montos generados estos son pequeños: aproximadamente hay un ingreso mensual de 300 soles, frente a sus egresos aún menores, es decir que logran cubrir sus obligaciones, sin embargo, alcanzan un nivel de bienestar limitado.

Así se aprecia que el $64,71 \%$ de la población llega tener egresos inferiores a los 900 nuevos soles, esto es explicado por que muchos de los alimentos son extraídos de los campos, su dieta es básicamente a partir de productos de la zona, cuentan con animales menores de los cuales disponen, y finalmente los servicios en el hogar no están presentes por lo que no representan carga de egresos (Tabla 9). 
Tabla 5: Características de la vivienda: material predominante en paredes, techos y pisos

\begin{tabular}{|c|c|c|c|c|c|c|}
\hline \multicolumn{2}{|c|}{ Material predominante en los techos } & Teja & $\begin{array}{c}\text { Calamina de } \\
\text { metal }\end{array}$ & $\begin{array}{c}\text { Calamina de } \\
\text { metal }\end{array}$ & $\begin{array}{l}\text { Calamina de } \\
\text { plástico }\end{array}$ & Concreto \\
\hline \multicolumn{2}{|c|}{ Material predominante en los pisos } & $\begin{array}{c}\text { Tierra / } \\
\text { arena }\end{array}$ & $\begin{array}{c}\text { Tierra / } \\
\text { arena }\end{array}$ & $\begin{array}{c}\text { Cemento / } \\
\text { ladrillo }\end{array}$ & Tierra / arena & $\begin{array}{c}\text { Tierra / } \\
\text { arena }\end{array}$ \\
\hline \multirow{6}{*}{$\begin{array}{l}\text { Material en } \\
\text { las paredes }\end{array}$} & & $\%$ & $\%$ & $\%$ & $\%$ & $\%$ \\
\hline & Adobe/ Tapial & 5,88 & 55,88 & 2,94 & 5,88 & 0,00 \\
\hline & Champas & 0,00 & 0,00 & 0,00 & 0,00 & 2,94 \\
\hline & Piedra & 0,00 & 2,94 & 0,00 & 0,00 & 0,00 \\
\hline & Madera / Quincha & 0,00 & 11,76 & 5,88 & 0,00 & 0,00 \\
\hline & Adobe/ Tapial & 0,00 & 5,88 & 0,00 & 0,00 & 0,00 \\
\hline
\end{tabular}

Fuente: Plan de Desarrollo Económico Local Motupe 2010

Tabla 6: Servicios de la vivienda

\begin{tabular}{|c|c|c|c|c|c|c|}
\hline \multicolumn{3}{|c|}{ Características de los servicios en la vivienda } & \multicolumn{3}{|c|}{ Tipo de Baño } & \multirow{3}{*}{$\begin{array}{c}\begin{array}{c}\mathrm{N}^{0} \text { total de } \\
\text { habitaciones }\end{array} \\
\text { Promedio }\end{array}$} \\
\hline & & & $\begin{array}{l}\text { Baño con } \\
\text { tasa }\end{array}$ & $\begin{array}{l}\text { Pozo } \\
\text { séptico }\end{array}$ & Otros & \\
\hline & & & $\%$ & $\%$ & $\%$ & \\
\hline \multirow{2}{*}{ Vivienda Propia } & \multirow{4}{*}{$\begin{array}{c}\text { Servicios } \\
\text { Higiénicos } \\
\text { dentro de la } \\
\text { casa: }\end{array}$} & $\mathrm{Si}$ & 2,94 & 2,94 & 0,00 & 4,50 \\
\hline & & No & 0,00 & 26,47 & 23,53 & 3,37 \\
\hline \multirow{2}{*}{$\begin{array}{l}\text { De los padres/ } \\
\text { suegros }\end{array}$} & & $\mathrm{Si}$ & 0,00 & 0,00 & 0,00 & . \\
\hline & & No & 0,00 & 2,94 & 0,00 & 2,00 \\
\hline
\end{tabular}

Fuente: Plan de Desarrollo Económico Local Motupe (2010).

Tabla 7: Tipo de energía dentro de la vivienda

\begin{tabular}{ccccccc}
\hline & & \multicolumn{5}{c}{ Energía usada para el alumbrado de la vivienda } \\
\cline { 3 - 7 } & & Kerosene & Vela/ cera & Luz eléctrica & Gas & Otro \\
& $\%$ & $\%$ & $\%$ & $\%$ & $\%$ \\
\hline \multirow{3}{*}{ Vivienda } & Propia & 0 & 71,875 & 3,125 & 0 & 25 \\
& Alquilada & 0 & 0 & 0 & 0 & 0 \\
& Prestada & 0 & 0 & 0 & 0 & 0 \\
& De los padres/ & 0 & 100 & 0 & 0 & 0 \\
\hline
\end{tabular}

Fuente: Plan de Desarrollo Económico Local Motupe (2010).

Tabla 8: Ingresos totales de la familia

\begin{tabular}{lcc}
\hline & Ingreso Promedio Anual* & Aporte $\%$ \\
\hline Jefe de Familia & 4176,92 & 81,69 \\
Cónyuge & 1625,00 & 9,78 \\
hijo/ familiar & 1425,00 & 4,29 \\
Miembro de familia & 1410,00 & 4,24 \\
Total & 3165,24 & 100,00 \\
\hline
\end{tabular}

*Sobre la base de miembros de la familia que manifiestan que tienen ingresos.

Fuente: Plan de Desarrollo Económico Local Motupe (2010). 
Tabla 9: Relación ingreso /gasto de la familia

\begin{tabular}{llcc}
\hline & & \multicolumn{2}{c}{ Ingresos Anuales 2012 } \\
\cline { 3 - 4 } Gastos Anuales 2012 & Promedio & $\%$ \\
& Menor de S/300 Soles & 5155,00 & 23,53 \\
& De S/. 301 a S/600 Soles & 4287,14 & 20,59 \\
& De S/. 601 a S/900 Soles & 1354,29 & 20,59 \\
& De S/. 901 a S/1500 Soles & 6000,00 & 2,94 \\
& De S/. 2001 a S/3900 Soles & 2775,00 & 2,94 \\
& No contestó & - & 29,42 \\
\hline
\end{tabular}

Fuente: Plan de Desarrollo Económico Local Motupe (2010).

El $20,59 \%$ tiene un gasto promedio anual que oscila entre S/ 601 y 900 nuevos soles, con un ingreso promedio anual de $\mathrm{S} /$ 1354 Soles, se podría entonces asumir que la población tiene algún tipo de capacidad de enfrentar inversiones y poder crecer, pero esto es relativo, ya que no contabilizan el pago de la mano de obra de los participantes (Tabla 10).

La extensión máxima de uso de la tierra para la agricultura por cada familia no supera las 5has, en este sentido los productores con extensiones más grandes recurren al sistema financiero en donde se destaca que de un grupo de tres personas, que mencionaron haber recibido crédito en los últimos cinco años, montos de S/ 1000 a S/5000 mil soles.
Ninguno devolvió el crédito por motivos de catástrofe y bajos precios.

La zona que no ha logrado establecer actividades vinculadas al mercado, lo que limita su competitividad, en donde el carácter de mayor relevancia es la economía de subsistencia.

Es a partir de algunas experiencias puntuales que logran el acceso a oportunidades como el crédito, la colocación de sus productos y las prácticas comerciales, las mismas que solo han sido esporádicas.

El mayor valor radica en la organización y el desarrollo de Asprobos como organismo de crecimiento y desarrollo colectivo, cuyo fin es el cuidado del Bosque Seco y su riqueza natural (Tabla 11).

Tabla 10: Uso de la tierra, cultivo, extensión y pertenencia

\begin{tabular}{ccccc}
\hline & \multicolumn{4}{c}{ Pertenencia el lote } \\
\cline { 2 - 5 } & $\begin{array}{c}\text { Propia } \\
\text { Área } \\
\text { Total ha }\end{array}$ & $\begin{array}{c}\text { Alquilado } \\
\text { Área } \\
\text { Total ha }\end{array}$ & $\begin{array}{c}\text { De la comunidad } \\
\text { Área } \\
\text { Total ha }\end{array}$ & $\begin{array}{c}\text { Familiar } \\
\text { Área } \\
\text { Total ha }\end{array}$ \\
\hline Maíz & 2,75 & 0 & 4,75 & 1 \\
Menestra & 2,75 & 0 & 0,75 & 0,25 \\
Camote & 0,25 & 0 & 0 & 0 \\
Mamey & 0,125 & 0 & 0,25 & 0 \\
Mango & 1,375 & 0 & 0,25 & 0 \\
Naranja & 0,375 & 0 & 0 & 0,25 \\
Palto & 0,25 & 0 & 0,25 & 0 \\
Plátano & 0,49 & 0 & 0,25 & 0 \\
Yuca & 0,25 & 0 & 0,25 & 0 \\
Zapallo & 0,25 & 0 & 0 & \\
\hline
\end{tabular}

Fuente: Plan de Desarrollo Económico Local Motupe (2010). 
Tabla 11: Matriz de evaluación por indicadores en Choloque

\begin{tabular}{|c|c|c|c|c|}
\hline Aspectos a evaluar & Indicadores & $\begin{array}{l}\text { Definición de } \\
\text { Indicadores }\end{array}$ & $\begin{array}{l}\text { Unidad de } \\
\text { Medida }\end{array}$ & $\begin{array}{l}\text { Instrumento } \\
\text { de Captura }\end{array}$ \\
\hline $\begin{array}{l}\text { Nivel de Ingreso } \\
\text { Familiar y Principales } \\
\text { Rasgos Sociales }\end{array}$ & $\begin{array}{l}\text { Ingreso } \\
\text { económico } \\
\text { Gasto Total } \\
\text { Vivienda /Predio }\end{array}$ & $\begin{array}{l}\text { Valor monetario x } \\
\text { mes } \\
\text { Promedio mensual } \\
\text { de gasto } \\
\text { Características de la } \\
\text { vivienda } \\
\text { Acceso a los } \\
\text { servicios: Educación } \\
\text { / Salud }\end{array}$ & $\begin{array}{l}\text { S/ } 320 \\
\text { S/ } 190 \\
\text { Bajo }\end{array}$ & $\begin{array}{l}\text { Encuesta } \\
\text { Encuesta y } \\
\text { Entrevistas } \\
\text { Encuesta } \\
\text { Encuesta }\end{array}$ \\
\hline \multirow{3}{*}{$\begin{array}{l}\text { Nivel de desarrollo } \\
\text { empresarial }\end{array}$} & $\begin{array}{l}\text { Nivel de } \\
\text { transformación } \\
\text { Emprendimiento }\end{array}$ & $\begin{array}{l}\text { Calidad del producto } \\
\text { Formación de } \\
\text { empresas }\end{array}$ & $\begin{array}{l}\text { Medio } \\
1 \%\end{array}$ & $\begin{array}{l}\text { Entrevista } \\
\text { Encuesta }\end{array}$ \\
\hline & Rentabilidad sobre & Utilidad neta/ventas & Sin registro & Encuesta \\
\hline & $\begin{array}{l}\text { Financiamiento } \\
\text { Capacitación }\end{array}$ & $\begin{array}{l}\text { Centros de } \\
\text { financiamiento } \\
\text { Uso del } \\
\text { conocimiento }\end{array}$ & $\begin{array}{l}5 \\
\text { Medio }\end{array}$ & Entrevista \\
\hline $\begin{array}{l}\text { Aspectos medio } \\
\text { ambientales. Nivel } \\
\text { de Uso de Recursos } \\
\text { Forestales y Niveles } \\
\text { de Organización }\end{array}$ & $\begin{array}{l}\text { Usos del bosque } \\
\text { Responsabilidad } \\
\text { medioambiental } \\
\text { Organización }\end{array}$ & $\begin{array}{l}\text { Tipos de usos } \\
\text { Tipos de actividades } \\
\text { Número de } \\
\text { organismos }\end{array}$ & $\begin{array}{l}3 \\
\text { Medio } \\
8\end{array}$ & $\begin{array}{l}\text { Sesión } \\
\text { Grupal } \\
\text { Encuesta } \\
\text { Entrevista }\end{array}$ \\
\hline
\end{tabular}

Fuente: Plan de Desarrollo Económico Local Motupe 2010

Es importante destacar que para obtener los ingresos y egresos promedios mensuales se utilizaron además entrevistas con el fin de cerrar la información. En este aspecto cabe señalar que solo en los egresos se contabilizo los gastos que efectúan, aún sigue sin incluir el valor de la mano de obra. El desarrollo empresarial solo está presente a través de la experiencia de Asprobos y esta ha logrado grandes desarrollos sin embargo aún no llegan a desenvolverse en un sistema de comercialización dinámico. Sin embargo, esta es la oportunidad hacer empresa de aquellas actividades que hoy se realizan intuitivamente.

\section{Conclusiones}

La unidad familiar de El Choloque tiene una conformación diversa ya que hay algunos casos en donde solo se identifica un miembro, hasta casos en los que llegan a ocho miembros, el típico es el promedio conformado por cinco miembros; en su mayoría son padres de edades jóvenes y maduros, siendo el jefe de la familia el varón cuya principal actividad es la agricultura.

La calidad de los servicios con los que cuenta la vivienda son básicos sobre infraestructura donde predomina materiales de la zona, con pisos de tierra, sobre comodidades no las poseen, es decir no tienen artefactos ni mobiliario de interior, la energía eléctrica no está presente dentro ni fuera de la vivienda, el agua potable del mismo modo, mucho menos cuentan con desagüe. El servicio de transporte es escaso en la ruta Tongorrape - Choloque, sin embargo, ya ubicados en la carretera cuentan con mejores opciones lo que les permite un tránsito hacia Motupe. Los caminos que llevan a Choloque son de trocha y entre las viviendas en algunos casos existen caminitos forzados por el propio agricultor, cuyas distancias son grandes, 
notándose un grado alto de dispersión territorial

Frente a este escenario poco atractivo se encuentra a un poblador positivo frente a su entorno, dinámico, en busca de oportunidades, tal es así que le da mucha importancia al conocimiento y la capacitación, en todas las familias los niños y adolescentes estudian, es decir existe acceso a la educación primaria y secundaria, sin embargo, los centros de enseñanza están ubicados a 45 minutos en promedio de las viviendas a los cuales llegan caminando. Son los padres los que poseen en su gran mayoría solo nivel primario y hasta secundario incompleto

Sus principales actividades son la agricultura de la cual generan sus ingresos, la crianza de animales que es complementaria, actualmente para quienes son de Asprobos la producción de miel y derivados es una oportunidad, así como la elaboración de mermeladas principalmente de mamey, productos que son aprovechados a partir de la participación en programas de desarrollo económico.

En el campo brilla dos cultivos el maíz y el mango, que les permiten algún nivel de intercambio comercial, dentro de las labores para llevar el cultivo se ve el uso intensivo de la mano de obra, no están tecnificados, el uso de pesticidas es mínimo, los factores pueden ser diversos pero de la visita se presumen tres, en el orden de prioridad: ingresos económicos bajos, conciencia ecológica y falta de información. Sin embargo, existe predisposición para un manejo integrado del cultivo, teniendo como último recurso el control químico, básicamente aquellos de última generación en base a extractos de hongos y algas, utilizados en la certificación orgánica.

Un aspecto interesante en la zona es el rol que juega Asprobos, la Asociación e Protección de los Bosques Secos es dinamizador para quienes son parte, ya que también se nota que la mitad de la población no está vinculada a esta asociación. Asprobos sensibilizo y puso en marcha un plan de manejo forestal, es sin duda este aspecto un pilar de su desarrollo futuro: los lugareños hacen funciones de guardabosques y guías de montaña con las instrucciones impartidas por el PNUD y las universidades que realizan trabajos de investigación en el bosque, sin embargo actualmente enfrentan un problema potencial, el deterioro del bosque, se detectó la presencia de enfermedades, de estas aún falta reconocer algunas por lo que se requiere de un estudio especializado en este aspecto.

El bosque seco y su aprovechamiento racional es el recurso que podría impulsar el desarrollo de la CC de Tongorrape. El caserío de Choloque que es parte del sector El Cardo, y este con los sectores de Marripon, La Capilla, Pueblo Nuevo, El Arrozal y Leticia son la CC de Tongorrape, en este sentido lo que suceda en El Choloque puede ser un pívot para los demás sectores en cuanto a la sostenibilidad de las zonas de Bosques Secos en general, pero debiera asegurarse la calidad de vida del poblador, es decir que con ingresos de subsistencia, sin servicios ni mejora de las familias; el escenario que se vislumbra es la migración de su población a otras ciudades que le ofrezcan oportunidad.

El aprovechamiento de los recursos del bosque es aun deficiente, ya que no se han identificado nuevos usos a ciertos recursos, desarrollo de productos incorporando valor agregado, o finalmente potencializar la miel y sus derivados posibilitando un sistema de comercialización que haga rentable a esta actividad e incluya a una mayor proporción de la población.

Si bien es cierto que la población solo participa parcialmente en algunos tipos de organización, se observa la permanencia en actividad de las mismas, es decir que tienen mecanismo que les permiten crecer y consolidarse como la presencia 
de pobladores líderes cuya dedicación ha favorecido a toda la población.

Finalmente se concluye confirmando que la población puede vencer sus limitaciones a través de la organización y el desarrollo de emprendimientos y sobre aquellos ya realizados avanzar empresarialmente.

\section{Literatura citada}

Asprobos 2004. Plan de Manejo Forestal Comunitario para los Bosques Secos de El Choloque. Boletín Campesino $\mathrm{N}^{\circ} 2$.

Banco Central de Reserva del Perú. 2004. Región Lambayeque: Cifras y reflexiones para el debate. Encuentro Económico Región Lambayeque.

Diagnóstico de la Micro Región Motupe Olmos 1992.

Dirección Regional de Trabajo y Promoción del Empleo Lambayeque 2011. Estudio de Muestreo Agrícola Tongorrape. Febrero 2011

Eguren, F. 2005. La Agricultura de la Costa Peruana, Lima.

INEI. 1993. Censo Nacional Agropecuario. Clasificación de las Tierras del Perú 1982.

INEI. 2007 Lambayeque Resultados Definitivos de los Censos Nacionales 1972, 1981 y 1993”. /Censos Nacionales: XI de Población y VI de Vivienda - Sistema de Consulta de Datos con R+SP Plan. Disponible en http://censos.inei.gob.pe/ Censos2007/redatam/

INEI. 2007. Lambayeque: "Resultados Definitivos de los Censos Nacionales 1993 y 2005". / Censos Nacionales: XI de Vivienda

Llacsahuanga, D.; Venancio, M. 2011 Tesis Estudio Pre Factibilidad para la Instalación de una planta procesadora de Goma de Sapote con fines de exportación al mercado europeo. UNALM Lima 2011.

Plan de Desarrollo Económico Local Motupe 2010.

PNUD. 2005. Informe de Desarrollo Humano. Perú 2005. Hagamos de la Competitividad una oportunidad para todos, 2005 eI Informe de Desarrollo Humano. Perú2006.Hacia una descentralización con ciudadanía, 2006. 